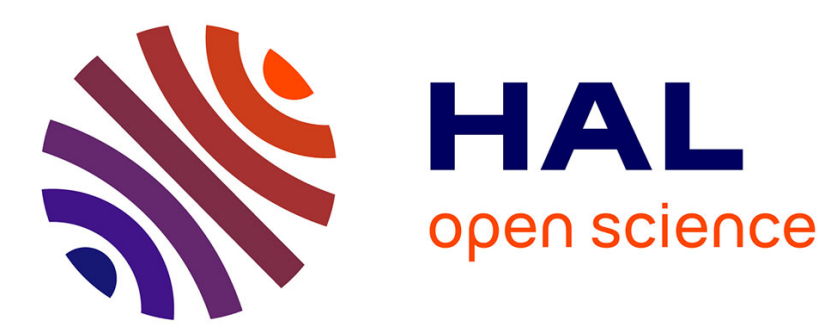

\title{
On the use of the Reciprocity-Gap functional in inverse scattering from planar cracks
}

Amel Ben Abda, Fabrice Delbary, Houssem Haddar

\section{To cite this version:}

Amel Ben Abda, Fabrice Delbary, Houssem Haddar. On the use of the Reciprocity-Gap functional in inverse scattering from planar cracks. [Research Report] RR-5290, INRIA. 2004, pp.23. inria00070710

\section{HAL Id: inria-00070710 \\ https://hal.inria.fr/inria-00070710}

Submitted on 19 May 2006

HAL is a multi-disciplinary open access archive for the deposit and dissemination of scientific research documents, whether they are published or not. The documents may come from teaching and research institutions in France or abroad, or from public or private research centers.
L'archive ouverte pluridisciplinaire HAL, est destinée au dépôt et à la diffusion de documents scientifiques de niveau recherche, publiés ou non, émanant des établissements d'enseignement et de recherche français ou étrangers, des laboratoires publics ou privés. 
INSTITUT NATIONAL DE RECHERCHE EN INFORMATIQUE ET EN AUTOMATIQUE

\section{On the use of the Reciprocity-Gap functional in inverse scattering from planar cracks}

Amel Ben Abda — Fabrice Delbary — Houssem Haddar

$\mathbf{N}^{\circ} \mathbf{5 2 9 0}$

Juin 2004

Thème NUM 



\title{
RINRIA
}

\section{On the use of the Reciprocity-Gap functional in inverse scattering from planar cracks}

\author{
Amel Ben Abda®, Fabrice Delbary \\ Thème NUM — Systèmes numériques \\ Projet Ondes
}

Rapport de recherche $\mathrm{n}^{\circ} 5290$ - Juin $2004-23$ pages

\begin{abstract}
We consider the inverse problem of determining the shape and location of planar screens inside a 3D body through acoustic or electromagnetic imaging. In order to determine the flaws we perform acoustic measurements with prescribed over-determined boundary data. The reciprocity gap concept is exploited to determine sound-hard planar screens of general shapes.
\end{abstract}

Key-words: Reciprocity Gap functional; inverse scattering; planar cracks recovering

\footnotetext{
* LAMSIN-Tunis

$\dagger$ INRIA-Rocquencourt
} 


\section{Utilisation de la fonctionnelle "Ecart à la Réciprocité" pour la recherche de fissures planes}

Résumé : On s'intéresse au problème de la détection de fissures planes dans un domaine $3 \mathrm{D}$ à l'aide d'ondes acoustiques ou électromagnétiques. En utilisant des données au bord surdéterminées et le concept d'écart à la réciprocité, on peut ainsi retrouver des fissures planes de forme quelconque.

Mots-clés : fonctionnelle "Ecart à la Réciprocité"; problème inverse en diffraction; recherche de fissures planes 


\section{Introduction}

In this contribution we consider the inverse crack problem defined by over-determined boundary data in the framework of the Helmholtz or Maxwell equations. Our concern focuses on the determination of planar cracks by means of a over-determined boundary data corresponding to electromagnetic time harmonic measurements. The inverse scattering problem for screens has been initiated by Kress in [13] where he shows, in particular, uniqueness results for sound soft two dimensional screens form the knowledge of scattered waves associated with all incident directions. The sound hard case is treated in by Mönch in [14. The case of Robin-like boundary condition has been considered by Alves-Ha-Duong in [1]. These uniqueness results can be easily generalized to three dimensional cases. For some uniqueness results using a finite number of measurements, we refer to the work of Rondi [15] and Cheng-Yamamoto [9]. Our contribution gives a uniqueness result for the particular case of planar screens with a constructive proof. There are, roughly speaking, two classes of screen identification. The first one consists in iterative processes which rely on multiple integration of the involved partial differential equation (Helmholtz and Maxwell in our case), and may be highly time consuming especially for 3D problems. These methods are, on the other hand, quite general and do not require strong assumptions on the screen geometry, such as flatness. Newton type methods have been numerically experimented in [13] and [1] for $2 \mathrm{D}$ problems. The second class of inversion procedures contains 'quasi-explicit' methods which do not require solving the forward problem at all and turns out to have quite low computational cost. The linear sampling method pertains to this class. It was originally developed for obstacles with non empty interior. It has been adapted to the case of screens by Brühl-Hanke [6] in the case of the electrostatic model and by Kirsch-Ritter [12, CakoniColton [7] and Cakoni-Colton-Darrigrandin [8] the electromagnetic case. This method is 'quasi-explicit' in so far as it does not require solving the forward problem at each step, but only involves the solution of an integral equation of the first kind with right-hand side dependent on some 'sampling point'. However its implementation requires a knowledge of the scattered waves for a large number of incident directions. The approach adopted here pertains to the second class of identification procedures. It makes use of the Reciprocity Gap concept introduced by Andrieux and Ben Abda [3, , [4] . When complete data are available on the boundary this tool turned out to be relevant for recovering 3D planar cracks in the case of elliptic equations (see [5] and references therein). The Reciprocity Gap concept allows the reconstruction of planar cracks in two steps.

(i) We first recover the host plane (I)

(ii) We then reconstruct the crack as the support of the solution jump across (П).

In these steps we make use of the so called Caldèron type fields as test functions for the reciprocity gap functional. Let us mention that these fields were also used to solve other types of inverse problems. We would like to mention for instance the work by Vogelius et al [2] on the reconstruction of small inhomogeneities and the work by Ikehata et al (see [11] and references therein) making use of these techniques for the reconstruction of the shape of a convex inclusion from Cauchy data.

The paper is outlined as follows: the opening section is devoted to the inverse acoustic

$\mathrm{RR} \mathrm{n}^{\circ} 5290$ 
problem. We adapt the Reciprocity Gap process to the case of sound-hard crack identification. In the third section we show the link between the Reciprocity Gap and the far field pattern which is usually used as data when identifying sound-hard or sound-soft screens in the free space. In the fourth section we treat the inverse problem of recovering planar cracks in the case of Maxwell's equations. The concluding section is devoted to the discussion of numerical experiments.

\section{The acoustic inverse problem}

We are concerned here with the reconstruction of a sound hard crack $\sigma$ from the knowledge of a scattered wave and its normal derivative at a fixed frequency on the boundary of a bounded and regular domain $\Omega$ that strictly contains $\sigma$. We shall restrict ourselves to plane cracks: $\sigma \subset \Pi$ where $\Pi$ is a plane (or a line in dimension 2), but not necessarily connected. The direct problem amounts to seek a solution $u \in H^{1}(\Omega \backslash \sigma)$ of

$$
\left\{\begin{array}{llll}
\text { (i) } & \Delta u+k^{2} u=0 & \text { in } & \Omega \backslash \sigma \\
\text { (ii) } & \partial_{n} u=0 & \text { on } & \sigma \\
\text { (iii) } & u=f & \text { on } & \partial \Omega
\end{array}\right.
$$

where $f \in H^{\frac{1}{2}}(\partial \Omega)$ is the given data and $k$ denotes the wave number. The well-posedness of this problem if $k^{2}$ is not an eigenvalue of the Laplace operator in $\Omega \backslash \sigma$ can be easily checked using a variational method, for instance. The inverse problem we are interested in is the reconstruction of the crack $\sigma$ from the knowledge of $f$ and the flux $g:=\partial_{n} u$ on $\partial \Omega$ where $n$ is the unit outward normal to $\partial \Omega$.

This reconstruction is based on the reciprocity gap functional $R G: H(\Omega) \mapsto \mathbb{C}$ defined by

$$
R G(v)=\int_{\partial \Omega} f \partial_{n} v d s-\int_{\partial \Omega} v g d s
$$

for all $v \in H(\Omega)$, where

$$
H(\Omega):=\left\{v \in H^{1}(\Omega) / \Delta v+k^{2} v=0 \text { in } \Omega\right\} .
$$

The integrals in (2) should be understood as a duality pairing between $H^{\frac{1}{2}}(\partial \Omega)$ and $H^{-\frac{1}{2}}(\partial \Omega$ ). This convention will be maintained without explicit mention throughout the remaining of the paper.

Applying the Green formula in $\Omega$ and using the boundary condition (1)ii) shows that

$$
R G(v)=\int_{\Pi}[u]_{\Pi} \nabla v \cdot \hat{N} d s \quad \text { for all } v \in H(\Omega),
$$

where $\hat{N}$ is a unitary normal vector to $\Pi$ and $[u]_{\Pi}:=u^{+}-u^{-}$on $\Pi$ where $u^{ \pm}(x)=$ $\lim _{\epsilon \rightarrow 0^{+}} u(x \pm \epsilon \hat{N})$ for a.e. $x \in \Pi$. We shall prove first that if $R G$ is not identically 0 then $\bar{\sigma}$ coincides with the support of the jump of $u$ across $\Pi$. 
Lemma 2.1 Assume that the host plane $\Pi$ is known and that the boundary data $f$ is such that $R G \neq 0$. Then $\operatorname{Supp}[u]_{\Pi}=\bar{\sigma}$.

Proof. The proof of this result follows the same idea as the proof of lemma 1.5 in [4] given for $k=0$. We present here a slight modification of that version. Without loss of generality one may assume that $\Pi=\left\{x \in \mathbb{R}^{3} ; x_{3}=0\right\}$. Let $V \subset \Omega$ be an open connected set containing $\sigma$, symmetric with respect to $\Pi$. Define

$$
\tilde{u}^{+}= \begin{cases}u^{+}\left(x_{1}, x_{2}, x_{3}\right) & x \in V^{+}, \\ u^{+}\left(x_{1}, x_{2},-x_{3}\right) & x \in V^{-},\end{cases}
$$

where $V^{+}=\{x \in V / x \cdot \hat{N}>0\}$ and $V^{-}=\{x \in V / x \cdot \hat{N}<0\}$.

Obviously

$$
\Delta \tilde{u}^{+}+k^{2} \tilde{u}^{+}=0 \text { in } V^{ \pm} .
$$

Now assume the existence of a part $\sigma_{0} \subset \sigma$ such that the surface measure of $\sigma_{0}$ is not 0 and such that $[u]_{\Pi}=0$ on $\sigma_{0}$. Let $V_{0}$ be an open connected subset of $V$ such that $V_{0} \cap \sigma \neq \emptyset$ and $\left(V_{0} \cap \sigma\right) \subset \sigma_{0}$. Since $[u]_{\Pi}=0$ and $\left[\partial_{N} u\right]_{\Pi}=0$ on $\sigma_{0}$, one has $\Delta u+k^{2} u=0$ in $V_{0}$, and also $\left[\tilde{u}^{+}\right]_{\Pi}=0$ and $\left[\partial_{N} \tilde{u}^{+}\right]_{\Pi}=0$ on $\sigma_{0}$. Therefore, $\Delta \tilde{u}^{+}+k^{2} \tilde{u}^{+}=0$ in $V_{0}$. Since $V_{0}$ is connected, the unique continuation principle ensures then that $u=\tilde{u}^{+}$in $V_{0}$ and the same principle ensures that $\tilde{u}^{+}=u^{-}$in $V^{-}$. Consequently, $[u]_{\Pi}=0$ on $\sigma$ and from (3) one gets that $R G=0$. This contradicts the hypothesis of the lemma.

Remark 2.1 The result of the previous lemma holds also if we assume that $\Pi$ is an analytic surface. The existence of $\tilde{u}^{+}$follows from classical theorems on analytic extension of solutions across analytic boundaries.

Thus, when the plane $\Pi$ is known, the crack $\sigma$ can be determined from a knowledge of $[u]_{\Pi}$. We shall now discuss the use of the reciprocity gap functional $R G$ to recover $\Pi$ and $[u]_{\Pi}$.

We shall denote by $\mathcal{F}$ the Fourier transform on $\Pi$ defined for $v \in L^{1}(\Pi)$ by

$$
\mathcal{F}(v)(\xi)=\int_{\Pi} v(x) \exp (i \xi \cdot x) d x \text { for all } \xi \in \mathbb{R}^{3}, \xi \cdot \hat{N}=0
$$

For $\theta \in \mathbb{C}^{3}$, we define

$$
v(x ; \theta)=\exp (i \theta \cdot x) \quad x \in \mathbb{R}^{3} .
$$

Simple calculations show that for $\theta$ such that $|\theta|=k, v(\cdot ; \theta) \in H(\Omega)$ and

$$
R G(v(\cdot ; \theta))=i(\theta \cdot \hat{N}) \int_{\sigma}[u]_{\Pi} \exp (i \theta \cdot x) d s(x)
$$

1) Recovering $[u]_{\Pi}$. We assume that $\Pi$ is known and is defined by the equation $x \cdot \hat{N}=\gamma$ where $\hat{N}$ is a unit normal to $\Pi$ and $\gamma$ is a given constant. From now on $\sqrt{ }$ denotes the complex square root with non negative imaginary part. Let $\xi \in \mathbb{R}^{3}, \xi \cdot \hat{N}=0$, and define

$$
\theta(\xi)=\xi-\sqrt{k^{2}-|\xi|^{2}} \hat{N} .
$$


Obviously $\theta(\xi) \cdot \theta(\xi)=k^{2}$ and therefore $v(\cdot ; \theta(\xi)) \in H(\Omega)$. Using (3), one easily verifies that

$$
R G(v(\cdot ; \theta(\xi)))=-i \sqrt{k^{2}-|\xi|^{2}} \exp \left(-i \gamma \sqrt{k^{2}-|\xi|^{2}}\right) \int_{\Pi}[u]_{\Pi} \exp (i \xi \cdot x) d x .
$$

Therefore

$$
\mathcal{F}\left([u]_{\Pi}\right)(\xi)=\frac{i \exp \left(i \gamma \sqrt{k^{2}-|\xi|^{2}}\right) R G(v(\cdot ; \theta(\xi)))}{\sqrt{k^{2}-|\xi|^{2}}} \text { for all } \xi \in \mathbb{R}^{3}, \xi \cdot \hat{N}=0,
$$

which explicitly determine $[u]_{\Pi}$ by taking the inverse Fourier transform of the right hand side.

2) Recovering the normal $\hat{N}$. Let $S$ be the unit sphere and define, for $\hat{\theta} \in S$,

$$
\Lambda(\hat{\theta})=\sup _{\varphi \in k S ; \varphi \perp \hat{\theta}}|R G(v(\cdot ; \varphi))| .
$$

Lemma 2.2 Assume that the boundary data $f$ is such that for each ellipse $\mathcal{E}$ centered at the origin of major axis $2 k$ and minor axis $\eta, 0 \leq \eta<2 k, \mathcal{F}\left([u]_{\Pi}\right)$ is not identically zero on $\mathcal{E}$. Then $\pm \hat{N}$ are the only zeros of $\Lambda$.

Proof. We first notice that $\Lambda( \pm \hat{N})=0$ since $R G(v(\cdot ; \varphi))=0$ for all $\varphi \perp \hat{N}$. Now assume the existence of $\hat{\theta} \in S$ such that $\hat{\theta} \neq \pm \hat{N}$ and $\Lambda(\hat{\theta})=0$. We deduce that $R G(v(\cdot ; \varphi))=0$ for all $\varphi \in k S$ such that $\varphi \cdot \hat{\theta}=0$.

$\mathcal{E}=\{\varphi-(\varphi \cdot \hat{N}) \hat{N} ; \varphi \in k S ; \varphi \perp \hat{\theta}\}$ is an ellipse centered at the origin of major axis $2 k$ and minor axis $2|\hat{\theta} \cdot \hat{N}|$. Since $\hat{\theta} \neq \pm \hat{N}, 2|\hat{\theta} \cdot \hat{N}|<2 k$. Hence $\mathcal{F}\left([u]_{\Pi}\right)$ is identically zero on $\mathcal{E} \backslash\left\{S_{1}, S_{2}\right\}$ where $S_{1}$ and $S_{2}$ are the endpoints of the major axis of $\mathcal{E}$. By continuity $\mathcal{F}\left([u]_{\Pi}\right)$ is zero on $\mathcal{E}$, which contradicts the hypothesis of the lemma.

Remark 2.2 The condition of the previous lemma is fulfilled if the boundary value $f$ is such that $\mathcal{F}\left([u]_{\Pi}\right)$ does not vanish on a connected path linking the origin to the circle of radius $k$ centered at the origin.

3) Recovering the plane $\Pi$. We assume here that $\hat{N}$ is known and shall determine the position of the plane $\Pi$. This amounts to the evaluation of the constant $\gamma=x \cdot \hat{N}$ for all $x \in \Pi$.

Lemma 2.3 Assume that $\hat{N}$ is known and that the boundary data $f$ is such that $R G \neq 0$. Then $\gamma$ is uniquely determined.

Proof. Since $\sigma \subset \Omega$ and $\Omega$ is bounded, one can obtain a priori lower and upper bounds $\gamma^{-}$ and $\gamma^{+}$of $\gamma$. Since $R G \neq 0,[u]_{\Pi} \neq 0$ and $\mathcal{F}\left([u]_{\Pi}\right) \neq 0$. Moreover, the support of $[u]_{\Pi}$ is compact, so $\mathcal{F}\left([u]_{\Pi}\right)$ is analytic on $\mathbb{R}^{2}$. Hence there exists $\theta \in k S$ such that

$$
0<\left(\gamma^{+}-\gamma^{-}\right)|\theta \cdot \hat{N}|<\pi \text { and } R G(v(\cdot, \theta)) \neq 0 .
$$

INRIA 
We set $\xi=\theta-(\theta \cdot \hat{N}) \hat{N}$ and $\theta^{\prime}=\xi-(\theta \cdot \hat{N}) \hat{N}$. Using (3) one get

$$
R G(v(\cdot, \theta))=c \exp (i \gamma(\theta \cdot \hat{N})) \text { and } R G\left(v\left(\cdot, \theta^{\prime}\right)\right)=-c \exp (-i \gamma(\theta \cdot \hat{N}))
$$

where $c=i(\theta \cdot \hat{N}) \int_{\Pi}[u]_{\Pi} \exp (i s \cdot \xi) d s$. The constant $\gamma$ is therefore uniquely determined in $\left[\gamma^{-}, \gamma^{+}\right]$by the ratio

$$
R G(v(\cdot, \theta)) / R G\left(v\left(\cdot, \theta^{\prime}\right)\right) .
$$

\section{Some remarks on the scattering problem}

We consider in this section the special case of scattering problems, i.e. when $\sigma$ is situated in free space. We would like to point out the link between $R G$ and the far field pattern that is commonly used as data in inverse scattering experiments like in [14]. We then describe in terms of incident plane waves the suitable choice of data for the inverse problem. Consider for instance $u \in H_{\text {loc }}^{1}\left(\mathbb{R}^{3} \backslash \sigma\right)$ satisfying

$$
\left\{\begin{array}{lll}
\Delta u+k^{2} u=0 & \text { in } & \mathbb{R}^{3} \\
\partial_{n} u=0 & \text { on } \quad \sigma
\end{array}\right.
$$

$u=u^{i}+u^{s}$ where $u^{i}(x)=\exp (i k x \cdot \hat{d})$ with $|\hat{d}|=1$ is an incident plane wave and where $u^{s}$ is the scattered wave satisfying the Sömmerfeld radiation condition

$$
\lim _{r=|x| \rightarrow \infty} r\left(\partial_{r} u^{s}-i k u^{s}\right)=0
$$

uniformly with respect to $\hat{x}=x / r$. It is well known [10] that the scattered field has the asymptotic expansion

$$
u^{s}(x)=\frac{e^{i k r}}{r}\left(u_{\infty}(\hat{x})+O(1 / r)\right)
$$

uniformly with respect to $\hat{x}$, where $u_{\infty}$ is called the far field pattern. On the other hand, from the representation theorem of a radiating solution to the Helmholtz equation [10], if $\Phi(x, y)$ denotes the fundamental solution given in dimension three by $\Phi(x, y)=\exp (i k \mid x-$ $y \mid) /(4 \pi|x-y|)$ for $x \neq y$, then the scattered field can be represented outside $\sigma$ by

$$
u^{s}(x)=\int_{\sigma}\left(\nabla_{y} \Phi(x, y) \cdot \hat{N}\right)[u]_{\Pi}(y) d y .
$$

From the asymptotic expansion

$$
\nabla_{y} \Phi(x, y)=\frac{\exp (i k|x|)}{4 \pi|x|}(-i k \hat{x} \exp (-i k \hat{x} \cdot y)+O(1 /|x|))
$$

$\mathrm{RR} \mathrm{n}^{\circ} 5290$ 
uniformly for all $y \in \sigma$, we derive

$$
u_{\infty}(\hat{x})=-\frac{i k}{4 \pi}(\hat{x} \cdot \hat{N}) \int_{\sigma}[u]_{\Pi}(y) \exp (-i k \hat{x} \cdot y) d y .
$$

Therefore, comparing this expression with (3) one gets

$$
u_{\infty}(\hat{x})=-\frac{R G(v(\cdot,-k \hat{x}))}{4 \pi}
$$

where $v(x, \theta)=\exp (i \theta \cdot x)$. One sees therefore the close link between the reciprocity gap functional and far field pattern. The identity (8) also indicates that the functional $R G$ contains more informations than the far field pattern and that is why it allows explicit reconstructions in the case of plane cracks.

Moreover, in the case of scattering problem, one can make clear the assumptions in Lemmas 2.1 and 2.3 The condition $R G \neq 0$ holds if and only if the incident wave $u^{i}$ is such that $\hat{d} \cdot \hat{N} \neq 0$. Obviously, if $\hat{d} \cdot \hat{N}=0$ then $R G=0$. Now, assume $R G=0$, then $u_{\infty}=0$ and therefore $u^{s}=0$ by Rellich's lemma and the unique continuation principle. The condition $\partial_{n} u=0$ on the scatterer then implies $\partial_{n} u^{i}=0$ on $\sigma$ and therefore $\hat{d} \cdot \hat{N}=0$.

We were not able to link the assumption of lemma 2.2 to some condition on the incident plane wave.

Remark 3.1 We would like to notice here that the use of the reciprocity gap functional to obtain explicit reconstructions applies in principle to more general boundary conditions on $\sigma$, for example of impedance type $\partial_{n} u^{ \pm} \pm i k \lambda^{ \pm} u^{ \pm}=0$ on $\sigma$ where $\lambda^{ \pm}$are the impedance of each side of the crack. The theoretical and numerical justification of this case are under study.

\section{Maxwell's equations}

We treat in this section the case of Maxwell's equations where $\sigma$ represents a screen. All the results of section 2 can be derived in a very similar manner. We denote

$$
H(\operatorname{curl}, \Omega):=\left\{E \in L^{2}(\Omega)^{3} ; \operatorname{curl} E \in L^{2}(\Omega)^{3}\right\}
$$

and if $\operatorname{div}_{s}$ denotes the surface divergence operator on $\partial \Omega$ and $n$ the normal to $\partial \Omega$ directed to the exterior of $\Omega$, then we define

$$
\begin{gathered}
H^{-\frac{1}{2}}(\operatorname{div}, \partial \Omega):=\left\{E \in H^{-\frac{1}{2}}(\partial \Omega)^{3} ; E \cdot n=0 ; \operatorname{div}_{s} E \in H^{-\frac{1}{2}}(\partial \Omega)\right\} \\
H^{-\frac{1}{2}}(\operatorname{curl}, \partial \Omega):=\left\{E \in H^{-\frac{1}{2}}(\partial \Omega)^{3} ; E \cdot n=0 ; \operatorname{div}_{s}(E \times n) \in H^{-\frac{1}{2}}(\partial \Omega)\right\}
\end{gathered}
$$

The electric field $E(x)$, which is a vector in $\mathbb{R}^{3}$, satisfies $E \in H(\operatorname{curl}, \Omega)$ and

$$
\left\{\begin{array}{llll}
(i) & \operatorname{curlcurl} E-k^{2} E=0 & \text { in } & \Omega \backslash \sigma \\
(\text { ii }) & E \times \hat{N}=0 & \text { on } & \sigma \\
\text { (iii) } & E \times n=F & \text { on } & \partial \Omega
\end{array}\right.
$$


where $F \in H^{-\frac{1}{2}}(\operatorname{div}, \partial \Omega)$ is the boundary data and $n$ is the normal to $\partial \Omega$ directed to the exterior of $\Omega$. The direct problem has been studied in [7] when it corresponds to a scattering problem, i.e. the first equation in (9) is satisfied in $\mathbb{R}^{3} \backslash \sigma$ with $E \times \hat{N}=0$ on $\sigma, E=E^{i}+E^{s}$; $E^{i}$ is an entire solution to Maxwell's equations and $E^{s}$ is the scattered field that satisfies the Silver-Müller radiation condition:

$$
\lim _{|x| \rightarrow \infty}\left(\operatorname{curl} E^{s} \times x-i k|x| E^{s}\right)=0
$$

uniformly in all directions $\hat{x}$. It is shown in [8] that there exists a unique solution $E$ in $H_{\text {loc }}\left(\right.$ curl, $\left.\mathbb{R}^{3}\right)$. The well-posedness of problem (9) if $k^{2}$ is not an eigenvalue of the Maxwell operator in $\Omega \backslash \sigma$ can be derived in a very similar way. We use as additional data for the inverse problem the boundary values

$$
G:=(\operatorname{curl} E)_{T} \quad \text { on } \partial \Omega,
$$

where $(V)_{T}$ denotes the tangential part of a surface vector field $V$. The reciprocity gap functional $R G_{M}$ is now defined on the space

$$
H_{M}(\Omega):=\left\{V \in H(\operatorname{curl}, \Omega) / \operatorname{curl} \operatorname{curl} V-k^{2} V=0 \text { in } \Omega\right\},
$$

by

$$
R G_{M}(V)=-\int_{\partial \Omega} F \cdot(\operatorname{curl} V)_{T} d s+\int_{\partial \Omega}(V \times n) \cdot G d s
$$

where the integrals should be understood as duality pairing between $H^{-\frac{1}{2}}(\operatorname{div}, \partial \Omega)$ and $H^{-\frac{1}{2}}(\operatorname{curl}, \partial \Omega)$ (this convention will be kept in the remaining of this section). From the Stokes formula and the boundary condition (9 ii), one derives

$$
R G_{M}(V)=\int_{\sigma}\left[(\operatorname{curl} E)_{T}\right]_{\Pi} \cdot(V \times \hat{N}) d s \text { for all } V \in H_{M}(\Omega) .
$$

We shall prove first that if $R G_{M}$ is not identically 0 then $\bar{\sigma}$ coincides with the support of the jump of $(\operatorname{curl} E)_{T}$ across $\Pi$.

Lemma 4.1 Assume that $\Pi$ is known and that the boundary data $F$ is such that $R G_{M} \neq 0$. Then $\operatorname{Supp}\left[(\operatorname{curl} E)_{T}\right]_{\Pi}=\bar{\sigma}$.

Proof. The proof of this result follows the same lines as the proof Lemma 2.1. Without loss of generality one may assume that $\left.\Pi=\left\{x \in \mathbb{R}^{3} ; x_{3}=0\right\}\right\}$. Let $E_{1}, E_{2}, E_{3}$ be the cartesian components of E. Let $W \subset \Omega$ be an open connected set containing $\sigma$, symmetric with respect to $\Pi$. Define

$$
\tilde{E}^{+}= \begin{cases}E^{+}\left(x_{1}, x_{2}, x_{3}\right) & x \in W^{+}, \\ \left(-E_{1}^{+}\left(x_{1}, x_{2},-x_{3}\right),-E_{2}^{+}\left(x_{1}, x_{2},-x_{3}\right), E_{3}^{+}\left(x_{1}, x_{2},-x_{3}\right)\right) & x \in W^{-} .\end{cases}
$$


Obviously

$$
\operatorname{curl} \operatorname{curl} \tilde{E}^{+}-k^{2} \tilde{E}^{+}=0 \text { in } W^{ \pm} .
$$

Now assume the existence of a part $\sigma_{0} \subset \sigma$ such that the surface measure of $\sigma_{0}$ is not 0 and such that $\left[(\operatorname{curl} E)_{T}\right]_{\Pi}=0$ on $\sigma_{0}$. Let $W_{0}$ be an open connected subset of $W$ such that $W_{0} \cap \sigma \neq \emptyset$ and $\left(W_{0} \cap \sigma\right) \subset \sigma_{0}$. Since $\left[(\operatorname{curl} E)_{T}\right]_{\Pi}=0$ and $\left[(E)_{T}\right]_{\Pi}=0$ on $\sigma_{0}$, one has curl curl $E-k^{2} E=0$ in $W_{0}$ and also $\left[\left(\operatorname{curl} E^{+}\right)_{T}\right]_{\Pi}=0$ and $\left[\left(E^{+}\right)_{T}\right]_{\Pi}=0$ on $\sigma_{0}$. Therefore, curl curl $\tilde{E}^{+}-k^{2} \tilde{E}^{+}=0$ in $W_{0}$. Since $W_{0}$ is connected, the unique continuation principle ensures then that $E=\tilde{E}^{+}$in $W_{0}$ and the same principle ensures that $\tilde{E}^{+}=E$ in $W^{-}$. Consequently, $\left[(\operatorname{curl} E)_{T}\right]_{\Pi}=0$ on $\sigma$ and from (11) one gets that $R G_{M}=0$, which is is contradiction with the hypothesis of the lemma.

As in the case of the Helmholtz equation, using some special test functions $V$ one can exploit (11) to recover the plane $\Pi$ and $\left[(\operatorname{curl} E)_{T}\right]_{\Pi}$. For $(\theta, \hat{p}) \in \mathbb{C}^{3} \times \mathbb{R}^{3}$ a directionpolarization couple, we define

$$
V(x ; \theta, \hat{p}):=\hat{p} \exp (i \theta \cdot x) \quad x \in \mathbb{R}^{3} .
$$

For $\theta \cdot \theta=k^{2}$ and $\hat{p} \cdot \theta=0, V(\cdot ; \theta, \hat{p})$ satisfies Maxwell's equations and therefore $V(\cdot ; \theta, \hat{p}) \in$ $H_{M}(\Omega)$. We also have that $\operatorname{curl} V(\cdot ; \theta, \hat{p}) \in H_{M}(\Omega)$.

1) Recovering $\left[(\operatorname{curl} E)_{T}\right]_{\Pi}$. We assume here that $\Pi$ is known and is defined by the equation: $x \cdot \hat{N}=\gamma$ where $\hat{N}$ is a unit normal to $\Pi$ and $\gamma$ is a given constant. Let $\xi \in \mathbb{R}^{3}$, $\xi \neq 0, \xi \cdot \hat{N}=0$, and define

$$
\theta(\xi)=\xi-\sqrt{k^{2}-|\xi|^{2}} \hat{N} \text { and } \hat{p}(\xi)=\hat{N} \times \hat{\xi}
$$

where $\hat{\xi}=\xi /|\xi|$. Then the couple $(\theta(\xi), \hat{p}(\xi))$ satisfies $\theta \cdot \theta=k^{2}$ and $\hat{p} \cdot \theta=0$. Since $\hat{p}(\xi) \times \hat{N}=\hat{\xi}$, one deduces from (11) that

$$
\begin{array}{r}
R G_{M}(V(\cdot ; \theta(\xi), \hat{p}(\xi)))=\exp \left(-i \gamma \sqrt{k^{2}-|\xi|^{2}}\right) \\
\int_{\Pi}\left(\left[(\operatorname{curl} E)_{T}\right]_{\Pi} \cdot \hat{\xi}\right) \exp (i \xi \cdot s) d s
\end{array}
$$

On the other hand, since $\xi \cdot \hat{N}=0$,

$$
\theta(\xi) \times \hat{p}(\xi)=|\xi| \hat{N}+\sqrt{k^{2}-|\xi|^{2}} \hat{\xi}
$$

Hence, $(\theta(\xi) \times \hat{p}(\xi)) \times \hat{N}=\sqrt{k^{2}-|\xi|^{2}} \hat{\xi} \times \hat{N}$ and

$$
\begin{gathered}
R G_{M}(\operatorname{curl} V(\cdot ; \theta(\xi), \hat{p}(\xi)))=i \sqrt{k^{2}-|\xi|^{2}} \exp \left(-i \gamma \sqrt{k^{2}-|\xi|^{2}}\right) \\
\int_{\Pi}\left(\left[(\operatorname{curl} E)_{T}\right]_{\Pi} \cdot(\hat{\xi} \times \hat{N})\right) \exp (i \xi \cdot s) d s .
\end{gathered}
$$


For all $\xi \in \mathbb{R}^{3}, \xi \neq 0$ and $\xi \cdot \hat{N}=0$, the pair $(\hat{\xi}, \hat{\xi} \times \hat{N})$ forms an orthonormal basis of $\Pi$. Therefore, if one sets

$$
\mathcal{A}(\xi)=R G_{M}(V(\cdot ; \theta(\xi), \hat{p}(\xi))) \text { and } \quad \mathcal{B}(\xi)=R G_{M}(\operatorname{curl} V(\cdot ; \theta(\xi), \hat{p}(\xi)))
$$

it follows from (12) and (13) that

$$
\mathcal{F}\left(\left[(\operatorname{curl} E)_{T}\right]_{\Pi}\right)(\xi)=\exp \left(i \gamma \sqrt{k^{2}-|\xi|^{2}}\right)\left(\mathcal{A}(\xi) \hat{\xi}-i \frac{\mathcal{B}(\xi)}{\sqrt{k^{2}-|\xi|^{2}}}(\hat{\xi} \times \hat{N})\right) .
$$

This identity now provides then an explicit reconstruction of $\left[(\operatorname{curl} E)_{T}\right]_{\Pi}$ by taking the inverse Fourier transform of the right hand side. According to the previous Lemma, this allows us to reconstruct $\sigma$ if the boundary value $F$ is such that $R G_{M} \neq 0$.

2) Recovering the normal $\hat{N}$. Now let $S$ be the unit sphere and define for $\hat{p} \in S$,

$$
\Lambda(\hat{p})=\sup _{\theta \in k S ; \theta \perp \hat{p}}|R G(V(\cdot ; \theta, \hat{p}))| .
$$

Lemma 4.2 Assume that for each ellipse $\mathcal{E}$ centered at the origin, with major axis $2 k$ and minor axis $\eta, 0 \leq \eta<2 k, \mathcal{F}\left(\left[(\operatorname{curl} E)_{T}\right]_{\Pi}\right) \cdot w$ is not identically zero on $\mathcal{E}$ where $w$ is the direction of the major axis of $\mathcal{E}$. Then $\pm \hat{N}$ are the only zeros of $\Lambda$.

Proof. We first notice that $\Lambda( \pm \hat{N})=0$ since $R G(V(\cdot ; \theta, \hat{p}))=0$ for all $\theta \perp \hat{N}$. Now assume the existence of $\hat{p} \in S$ such that $\hat{p} \neq \pm \hat{N}$ and $\Lambda(\hat{p})=0$. From this we deduce that $R G(V(\cdot ; \theta, \hat{p}))=0$ for all $\theta \in k S$ such that $\theta \cdot \hat{p}=0$.

Therefore, for all $\theta \in k S$ such that $\theta \cdot \hat{p}=0, R G(V(\cdot ; \theta, \hat{p}))=0$. Hence, using (11) we have

$$
\exp \left(i(\theta \cdot \hat{N})\left(x_{0} \cdot \hat{N}\right)\right)\left(\int_{\Pi}\left[(\operatorname{curl} E)_{T}\right]_{\Pi} \exp (i x \cdot \xi) d x\right) \cdot(\hat{p} \times \hat{N})=0
$$

where $x_{0}$ is some point in $\Pi$.

$\mathcal{E}=\{\theta-(\theta \cdot \hat{N}) \hat{N} ; \theta \in k S ; \theta \perp \hat{p}\}$ is an ellipse centered at the origin of major axis $w=2 k \frac{\hat{p} \times \hat{N}}{\|\hat{p} \times \hat{N}\|}$ and of minor axis of length $2|\hat{p} \cdot \hat{N}| k<2 k$ (since $\hat{p} \neq \pm \hat{N}$ ). Moreover, (16) yields $\mathcal{F}\left(\left[(\operatorname{curl} E)_{T}\right]_{\Pi}\right) \cdot w=0$ on $\mathcal{E}$, which contradicts the hypothesis of the lemma.

3) Recovering the plane $\Pi$. We assume here that $\hat{N}$ is known and shall determine the position of the plane $\Pi$. This amounts to the evaluation of the constant $\gamma=x \cdot \hat{N}$ for all $x \in \Pi$.

Lemma 4.3 Assume that $\hat{N}$ is known and that the boundary data $F$ is such that $R G_{M} \neq 0$. Then $\gamma$ is uniquely determined.

Proof. Since $\sigma \subset \Omega$ and $\Omega$ is bounded, one can obtain a priori lower and upper bounds $\gamma^{-}$ and $\gamma^{+}$of $\gamma$. 
Since $R G_{M} \neq 0$, there exists $\theta \in k S$ and $\hat{p} \in S$ such that $R G(V(\cdot ; \theta, \hat{p})) \neq 0$. Therefore, $\left[(\operatorname{curl} E)_{T}\right]_{\Pi} \neq 0$ and $\mathcal{F}\left(\left[(\operatorname{curl} E)_{T}\right]_{\Pi}\right) \neq 0$. Moreover, the support of $\left[(\operatorname{curl} E)_{T}\right]_{\Pi}$ is compact, so $\mathcal{F}\left(\left[(\operatorname{curl} E)_{T}\right]_{\Pi}\right)$ is analytic on $\mathbb{R}^{2}$. Hence there exists $\theta \in k S$ such that

$$
0<\left(\gamma^{+}-\gamma^{-}\right)|\theta \cdot \hat{N}|<\pi \text { and } \mathcal{F}\left(\left[(\operatorname{curl} E)_{T}\right]_{\Pi}\right)(\xi) \neq 0
$$

where $\xi=\theta-(\theta \cdot \hat{N}) \hat{N}$.

Since $\mathcal{F}\left(\left[(\operatorname{curl} E)_{T}\right]_{\Pi}\right)(\xi) \neq 0$, there exists $w \in \mathbb{R}^{3}, w \cdot \hat{N}=0$ such that $\mathcal{F}\left(\left[(\operatorname{curl} E)_{T}\right]_{\Pi}\right)(\xi)$. $w \neq 0$. We then set $\hat{p}=\frac{\theta \times w}{\|\theta \times w\|}$. Thus we have $R G(V(\cdot ; \theta, \hat{p})) \neq 0$. We $\operatorname{set} \theta^{\prime}=\xi-(\theta \cdot \hat{N}) \hat{N}$. Using (11) one gets

$$
R G(V(\cdot ; \theta, \hat{p}))=c \exp (i \gamma(\theta \cdot \hat{N})) \text { and } R G\left(V\left(\cdot ; \theta^{\prime}, \hat{p}\right)\right)=c \exp (-i \gamma(\theta \cdot \hat{N}))
$$

where $c=\left(\int_{\Pi}\left[(\operatorname{curl} E)_{T}\right]_{\Pi} \exp (i s \cdot \xi) d s\right) \cdot(\hat{p} \times \hat{N})$. The constant $\gamma$ is therefore uniquely determined in $\left[\gamma^{-}, \gamma^{+}\right]$by the ratio

$$
R G(V(\cdot ; \theta, \hat{p})) / R G\left(V\left(\cdot ; \theta^{\prime}, \hat{p}\right)\right) .
$$

\section{Numerical implementation and results}

Only the acoustic case will be considered in this section. We use synthetic data that corresponds to the scattering of incident plane waves. The forward solver is based on reformulating the scattering problem as an integral equation using a double-layer potential. The resulting equation is discretized using $P^{1}$ edge elements.

\subsection{Numerical difficulties of the inverse scheme}

There are some numerical difficulties linked with the application of the inverse scheme described in section 2 The first one is associated with the determination of the normal to the host plane $\Pi$. The second difficulty arises with the determination of the normal to the plane crack $\Pi$. There is no a priori selection of the measurements (i.e. boundary data) that insures the assumption on $\mathcal{F}\left([u]_{\Pi}\right)$ required by lemma 2.2 However the sufficient condition given in remark 2.2 is in fact easier to check numerically. Since there is, so far, no theoretical link between this condition and the choice of the boundary data, the only systematic strategy is trial and error by varying the number $k$ or the boundary data $f$. In scattering problems, this trial and error would not be costly since numerical experiments showed that this condition holds for "all" frequencies $k$ and all incident directions $\hat{d}$ non orthogonal to $\hat{N}$. However, a better choice would be the use of low frequency $k$ (for example $k<2 \pi / D$ where $D$ is the diameter of the crack). This choice prevents high oscillations of $[u]_{\Pi}$ (see Figure 1) and therefore allows an easier determination of the zeros of the functional $\Lambda$. 
To recover the position of the plane one has to seek $\theta$ satisfying [5]. In scattering experiments one can establish a systematic choice of $\theta$ for any frequency if the direction $\hat{d}$ of the incident plane wave is well chosen. In particular, take this direction so that

$$
0<|\hat{d} \cdot \hat{N}|<\pi / k\left(\gamma^{+}-\gamma^{-}\right)
$$

Then $\theta=k \hat{d}$ satisfies the first requirement on condition (5). From (8) we have $R G(v(\cdot, \theta))=$ $4 \pi u_{\infty}(-\hat{d})$. From the optical theorem one has

$$
\Im\left(u_{\infty}(-\hat{d})\right)=\frac{k}{4 \pi}\left\|u_{\infty}\right\|_{L^{2}}^{2}
$$

which shows that $R G(v(\cdot, \theta)) \neq 0$ since $u_{\infty} \neq 0(\hat{d} \cdot \hat{N} \neq 0)$. Therefore $\theta=k \hat{d}$ satisfies the condition (5).

Let us notice that in practice it is more convenient to change the frequency and not the incident direction. As mentioned before, numerical experiments showed that $R G(v(\cdot, \theta)) \neq 0$ if $\theta$ is non orthogonal to $\hat{N}$. Now if we further ensure $k<\pi /\left(\gamma^{+}-\gamma^{-}\right)$then any $\theta$ non orthogonal to $\hat{N}$ would be convenient.

The first difficulty is linked to the evaluation of $\mathcal{F}\left([u]_{\Pi}\right)$ outside the disc of radius $k$. This difficulty derives from the ill-posedness of the inverse problem. More precisely, the evaluation of $\mathcal{F}\left([u]_{\Pi}\right)$ involves test fields $v(\cdot, \theta(\xi))$ with complex valued vectors $\theta(\xi)$. The numerical evaluation of $R G(v(\cdot, \theta(\xi)))$ is unstable because of the exponential factor $e^{-\sqrt{|\xi|^{2}-k^{2}}(x \cdot \hat{N})}$. In the following numerical trials we use a rough regularisation process which consists in the truncation of the Fourier transform by evaluating $\mathcal{F}\left([u]_{\Pi}\right)$ for $|\xi|<k$ and inverting it to obtain an approximation of $[u]_{\Pi}$.

\section{$5.2 \quad$ Numerical trials}

In implementing an identification algorithm based on measurements one has to keep in mind that measured data are subject to noise, the effects of which have to be studied. In the following numerical trials the data are synthetic, i.e. obtained by a boundary element computation subject to errors, and they are, hence, already noisy. To the computational noise, we have added a random noise generated by a Fortran routine. Notice that the shape reconstruction, that is the second step of our identification process, is not that sensitive to the amplitude of the noise (up to 50\%). Indeed the random noise is filtered when we evaluate $[u]_{\Pi}$ since this jump is calculated via an inverse Fourier transform.

A more realistic noise is considered by taking data associated with non perfectly flat cracks (see Figure 17).

For all the numerical trials, $\partial \Omega$ is taken to be the sphere of radius 1 centered at the origin.

To test the recovery of the host plane $\Pi$ we take as an example a square-shaped crack of side 1 in the plane (Oxy). Figure 1 illustrates the dependence of the Fourier transform of $[u]_{\Pi}$ on the wave number $k$. To retrieve the plane $\Pi$ we fixed $k=3$. The following table 
summarizes the results for different incident plane waves. The incident directions are given in terms of their spherical angles (using geophysical convention).

\begin{tabular}{||c|c|c||}
\hline $\begin{array}{c}\text { Incident } \\
\text { direction }\end{array}$ & $\begin{array}{c}\text { Normal } \\
\left(\text { exact }=(0,0,1)^{t}\right)\end{array}$ & $\begin{array}{c}\text { Position } \gamma \\
(\text { exact }=0)\end{array}$ \\
\hline$\left(90^{\circ}, 0^{\circ}\right)$ & $\left(0.565710^{-3},-0.913410^{-3}, 0.9999994\right)^{t}$ & $-0.00210^{-4}$ \\
\hline$\left(60^{\circ}, 60^{\circ}\right)$ & $\left(0.565710^{-3},-0.913410^{-3}, 0.9999994\right)^{t}$ & $-0.06910^{-4}$ \\
\hline$\left(30^{\circ}, 45^{\circ}\right)$ & $\left(-5.810610^{-3},-1.805210^{-3}, 0.9999815\right)^{t}$ & $-2.15710^{-4}$ \\
\hline
\end{tabular}
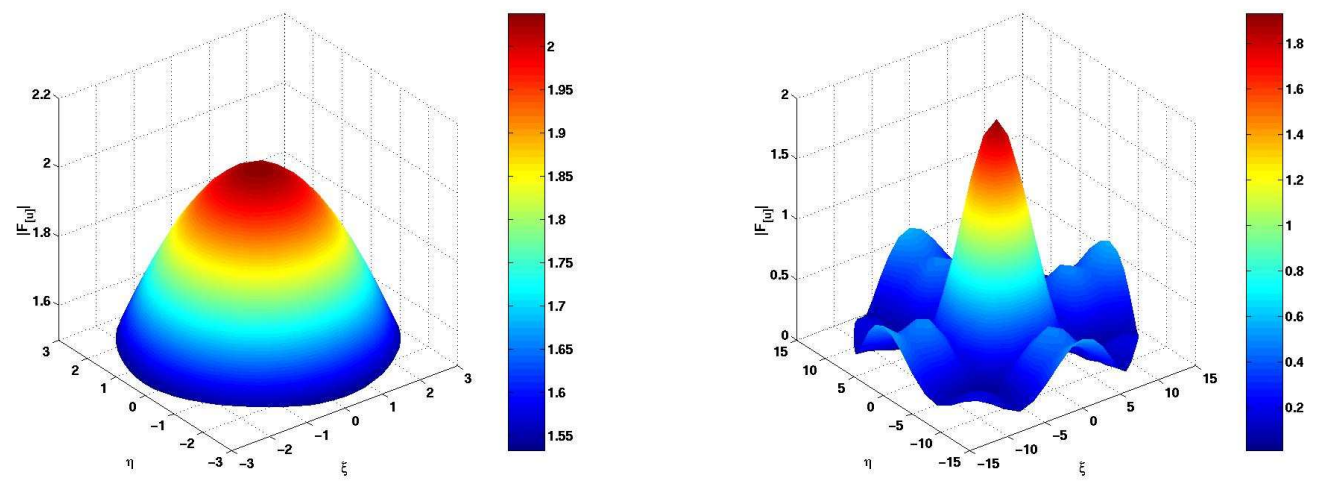

Figure 1: Modulus of the Fourier transform of $[u]_{\Pi}$ restricted to the disc of radius $\mathrm{k}$ (left $\mathrm{k}=$ 3 and right $\mathrm{k}=15)$. The incident direction is orthogonal to the crack.

Figure 2 illustrates how the reconstruction of the Fourier transform of $[u]_{\Pi}$ is unstable outside the disc of radius $k$. However, one gets a very accurate reconstruction inside that disc (compare with th right hand side of Figure 1). 

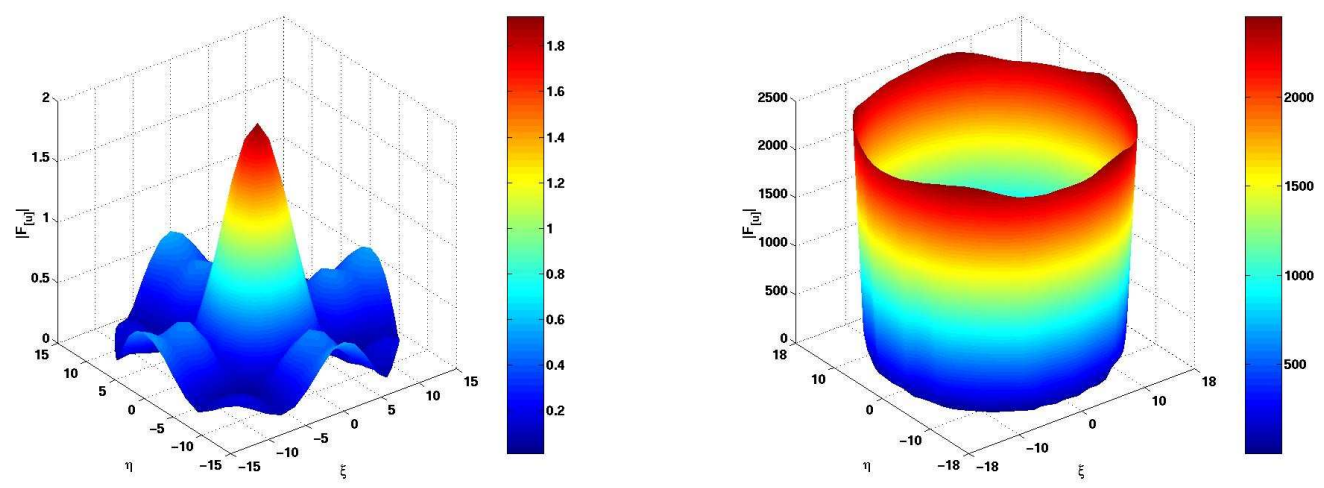

Figure 2: Modulus of the reconstructed Fourier transform of $[u]_{\Pi}$ restricted to the disc of radius $k=15$ (left) and restricted to the disc of radius $1.4 k$ (right).

As mentioned above, the complete identification of the crack is achieved by the reconstruction of $[u]_{\Pi}$. The jump of $u$ across $\Pi$ is approximated by taking the inverse Fourier transform of $\mathcal{F}\left([u]_{\Pi}\right)$ (evaluated via the RG functional) restricted to the disc of radius $k$.

To test the efficiency of our inversion process, we examine four numerical situations to recover an $\mathrm{X}$-shaped, a D-shaped and four squares planar cracks as well as an $\mathrm{X}$-shaped quasi-planar crack. These choices include a non connected geometry and a non simply connected one. We would like to mention that, up to our knowledge, there is no uniqueness result -except the one given in this work- for the case of sound-hard non simply connected cracks.

Our first numerical experiment corresponds to an X-shaped crack (see Figure 3). Figure 4 illustrates the reconstructions obtained for various wave numbers when the host plane is known. We used normal incident plane waves. As expected, the higher the frequency, the better is the reconstruction. Notice for instance that $k=40$ gives a quite good idea of the crack shape.

Figures 5 8 8 present experiments where the host plane is also unknown. In a first step we recover this plane using an (arbitrary) incident direction (here $\hat{d}=(1 / 4, \sqrt{3} / 4, \sqrt{3} / 2)$ and a small wavenumber, for instance $k=3$. Once the host plane is recovered we use a higher frequency $(k=40)$ to recover $[u]_{\Pi}$ and change the direction of the incident wave so that it coincides with the reconstructed normal to the host plane. In these figures we vary the amount of added random noise. In Figures [5.6, no random noise is added to recover the normal. However we added $50 \%$ of relative random noise in figure 6. We clearly observe that the reconstruction of $[u]_{\Pi}$ (that is the second part of our process) is robust. The first step is much more sensitive to added noise as shown in Figures 7.8 . The same conclusions hold for the D-shaped crack (Figures 9, 10, 11] and 12) as well as for the four squares crack (Figures 13, 14, 15] and 16). 


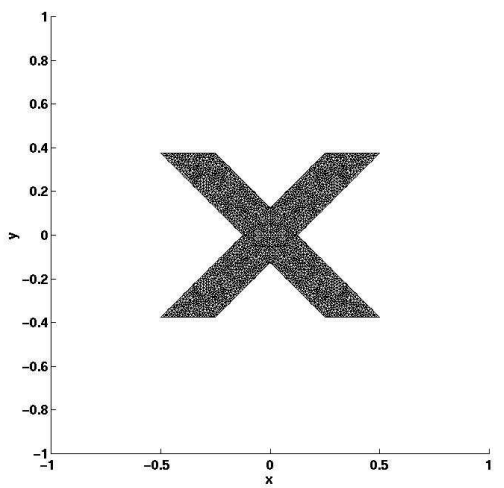

Figure 3: Mesh of the X-shaped crack. 

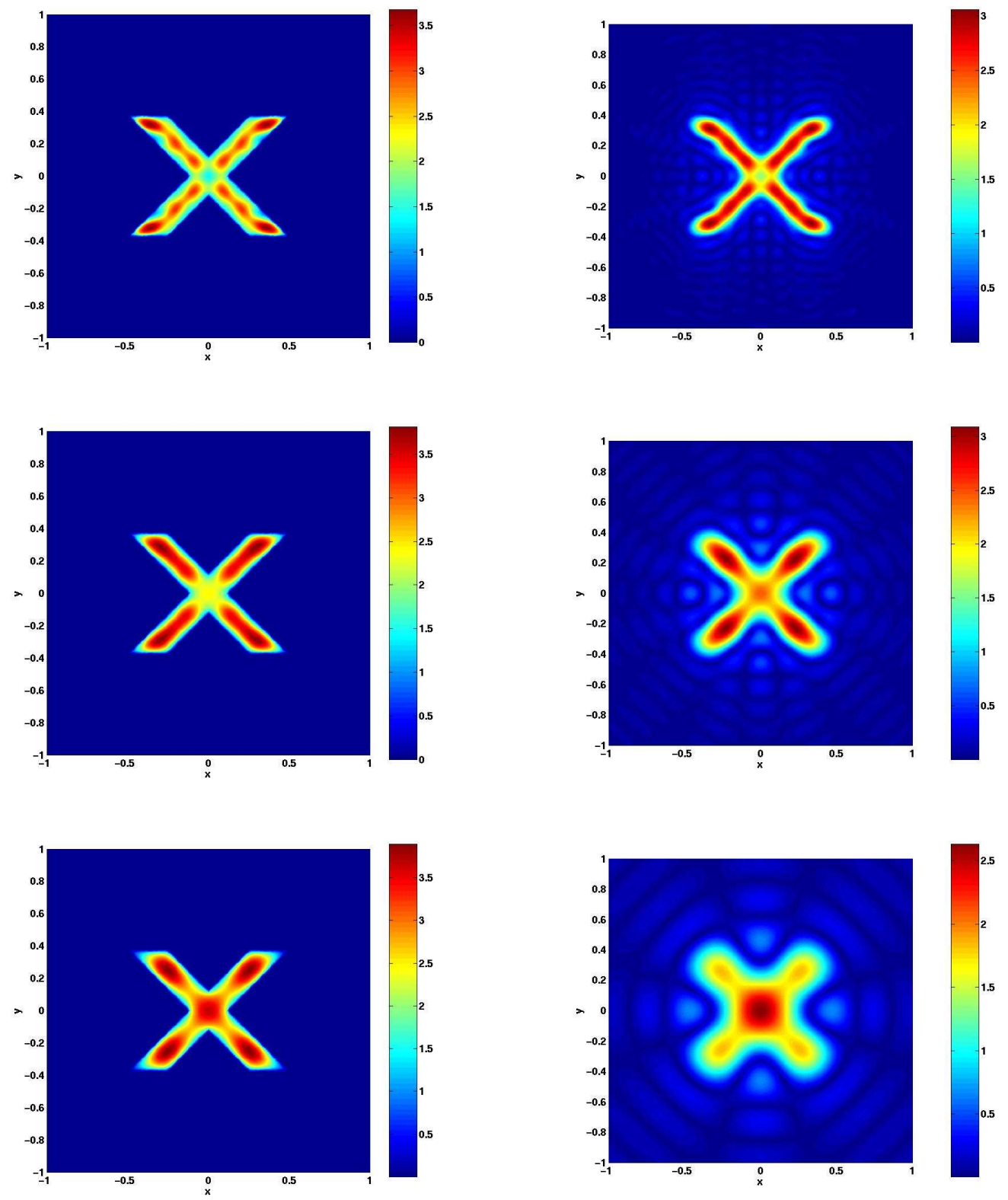

Figure 4: Modulus of $[u]_{\Pi}$, exact on the left hand side and (noise free) reconstructed on the right hand side. From the top to the bottom : $k=40, k=25, k=15$ (the host plane is known). 


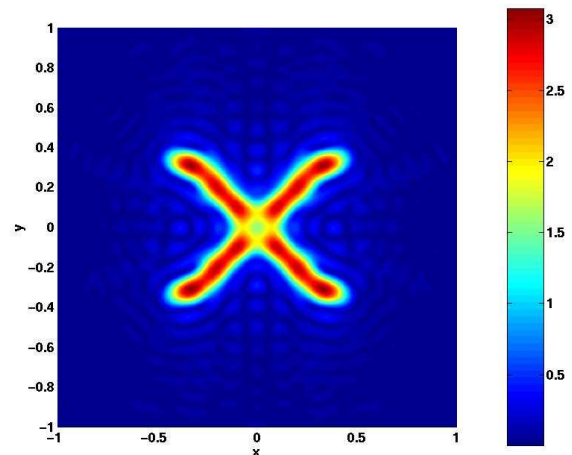

Figure 5: Modulus of reconstructed $[u]_{\Pi}$ with $0 \%$ noise on data to recover the host plane $(k=3)$ and $0 \%$ noise on data to recover $[u]_{\Pi}(k=40)$.

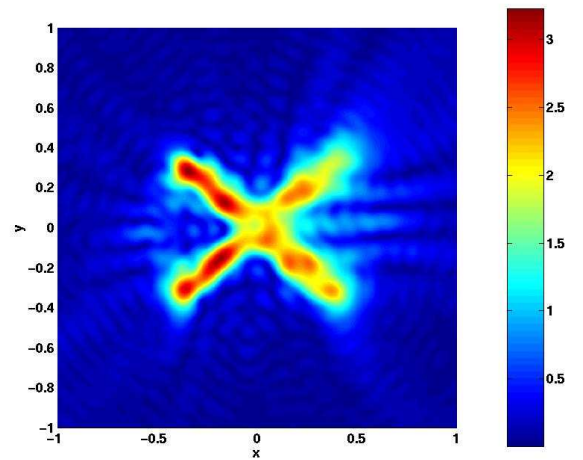

Figure 7: Modulus of reconstructed $[u]_{\Pi}$ with $10 \%$ noise on data to recover the host plane $(k=3)$ and $10 \%$ noise on data to recover $[u]_{\Pi}(k=40)$.

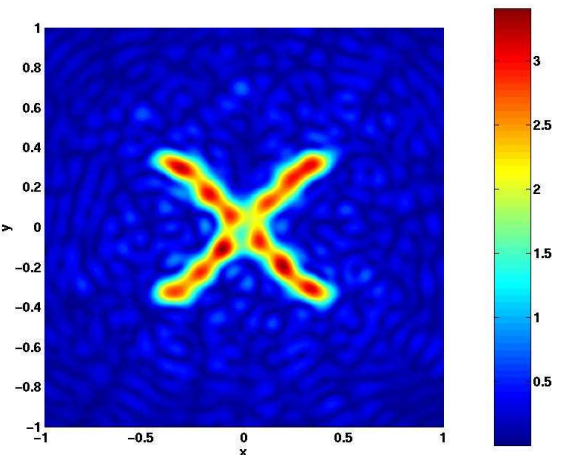

Figure 6: Modulus of reconstructed $[u]_{\Pi}$ with $0 \%$ noise on data to recover the host plane $(k=3)$ and $50 \%$ noise on data to recover $[u]_{\Pi}(k=40)$.

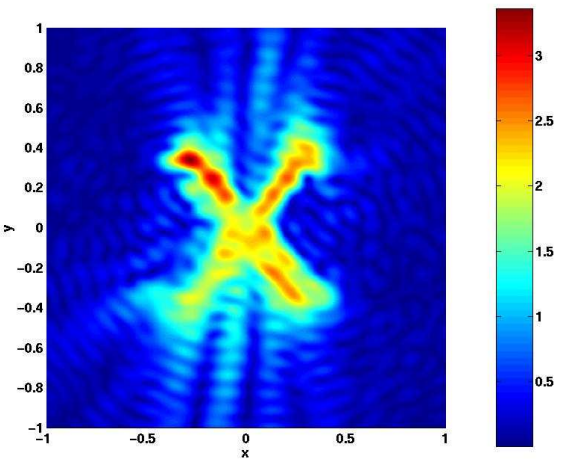

Figure 8: Modulus of reconstructed $[u]_{\Pi}$ with $25 \%$ noise on data to recover the host plane $(k=3)$ and $25 \%$ noise on data to recover $[u]_{\Pi}(k=40)$. 


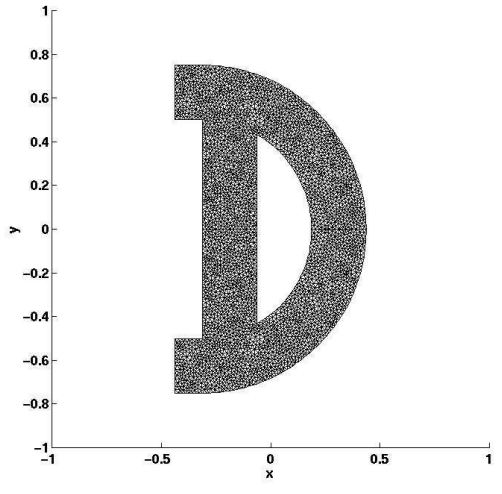

Figure 9: Mesh of the D-shaped crack.

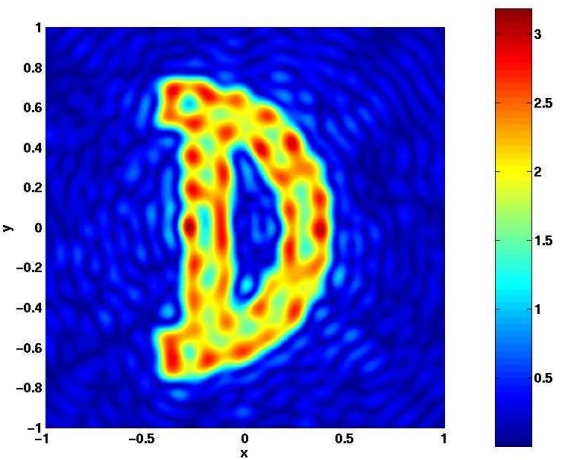

Figure 11: Modulus of reconstructed $[u]_{\Pi}$ with $0 \%$ noise on data to recover the host plane $(k=3)$ and $50 \%$ noise on data to recover $[u]_{\Pi}(k=40)$.

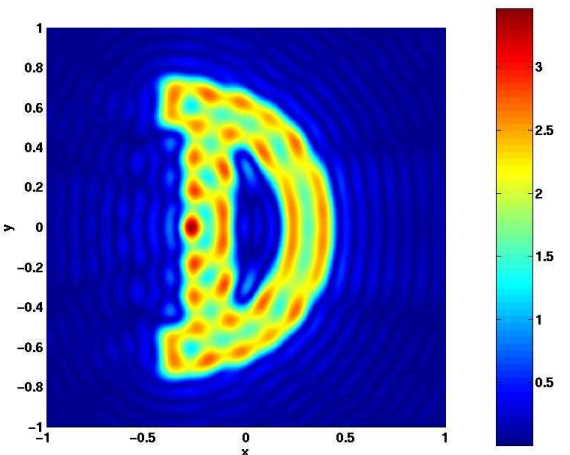

Figure 10: Modulus of reconstructed $[u]_{\Pi}$ with $0 \%$ noise on data to recover the host plane $(k=3)$ and $0 \%$ noise on data to recover $[u]_{\Pi}(k=40)$.

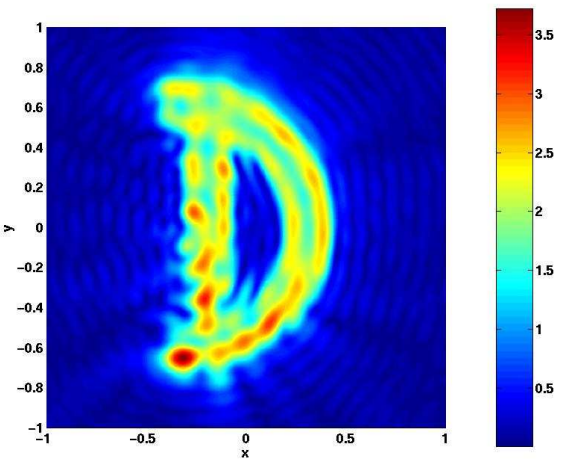

Figure 12: Modulus of reconstructed $[u]_{\Pi}$ with $10 \%$ noise on data to recover the host plane $(k=3)$ and $10 \%$ noise on data to recover $[u]_{\Pi}(k=40)$. 


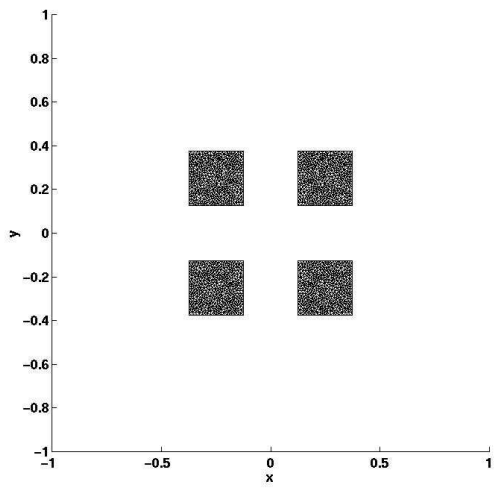

Figure 13: Mesh of the four square-shaped cracks.

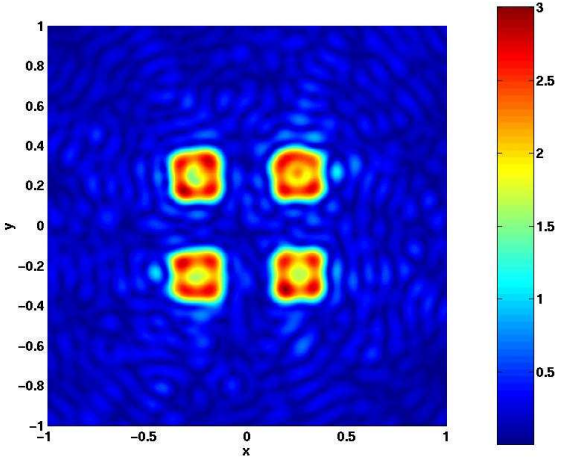

Figure 15: Modulus of reconstructed $[u]_{\Pi}$ with $0 \%$ noise on data to recover the host plane $(k=3)$ and $50 \%$ noise on data to recover $[u]_{\Pi}(k=40)$.

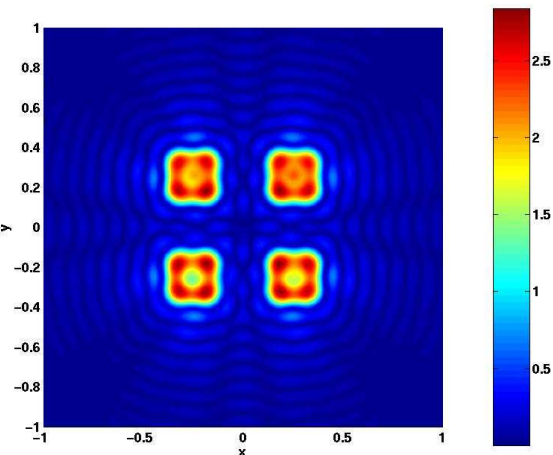

Figure 14: Modulus of reconstructed $[u]_{\Pi}$ with $0 \%$ noise on data to recover the host plane $(k=3)$ and $0 \%$ noise on data to recover $[u]_{\Pi}(k=40)$.

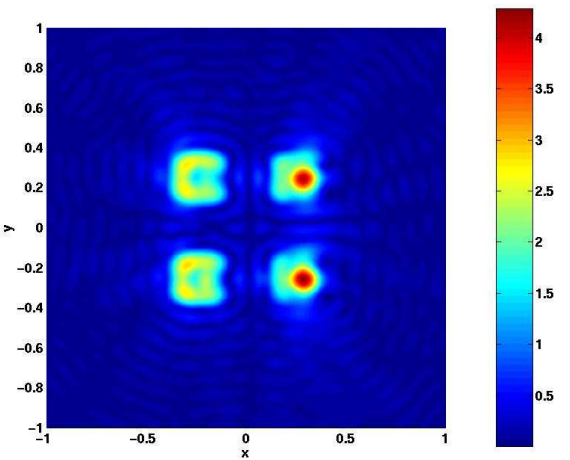

Figure 16: Modulus of reconstructed $[u]_{\Pi}$ with $10 \%$ noise on data to recover the host plane $(k=3)$ and $10 \%$ noise on data to recover $[u]_{\Pi}(k=40)$.

Non perfectly flat crack. We reconsider here the case of an X-shaped crack and would like to test the influence of the lack of flatness on our reconstruction process (see Figure 17). 
In this case our data would simulate in some sense a kind of realistic noisy data.

We use a wave of incidence $\left(60^{\circ}, 60^{\circ}\right)$ and of wavenumber $k=3$ to recover the plane $\Pi$. The application of our inverse scheme gave $\hat{N}=\left(-3.107550010^{-2}, 3.255498810^{-2}, 0.9989867\right)^{t}$ and a position $\gamma=2.745678610^{-3}$. Then the reconstruction of $[u]_{\Pi}$ was performed with $k=40$. The result is given in Figure 18 showing that we still have a good idea of the crack shape.
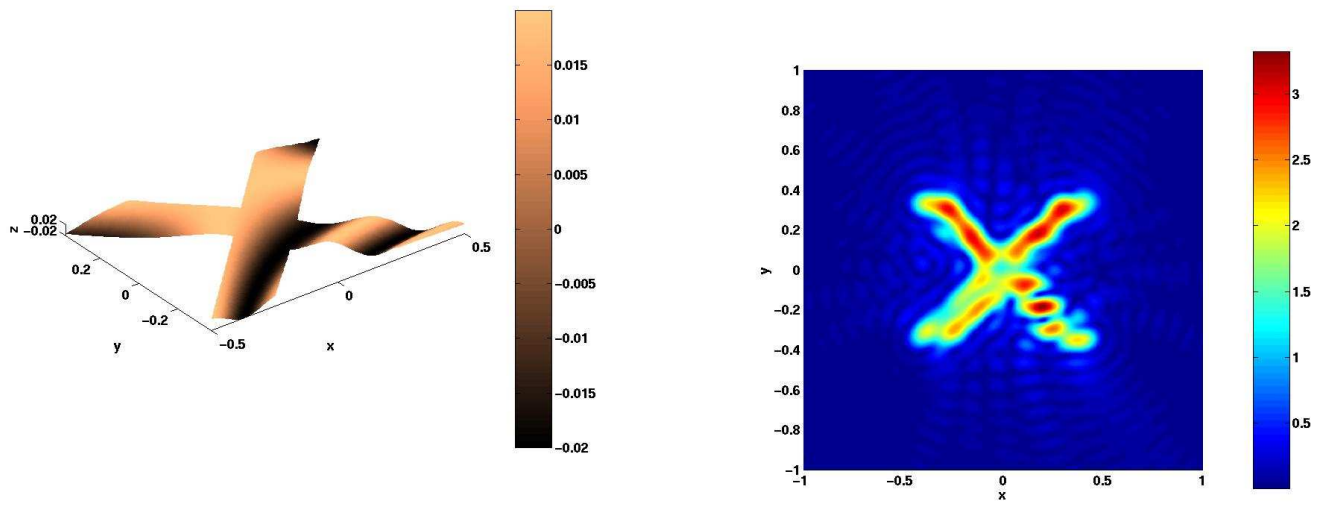

Figure 17: Perturbed X-shaped crack : $z(x, y)=0.02 \sin \left(2 \pi\left(2 x^{2}+3 x y-4 y^{2}-x+\right.\right.$ $2 y))$

\section{Acknowledgment}

Amel Ben Abda's work is supported by the Tunisian Secretary of State for Research, within the LAB-STI-02 Programme, and by INRIA through its associate teams programme eDIDON.

\section{References}

\section{References}

[1] Alves C., Ha Duong T. (1997): 'On inverse scattering by screens', Inverse Problems 13, 1161-1176 
[2] Ammari H., Moskow S., Vogelius M. (2003): 'Boundary integral formulae for the reconstruction of electric and electromagnetic inhomogeneities of small volume', ESAIM: Control, Optimisation and Calculus of Variations 9, 49-66

[3] Andrieux, S., Ben Abda, A. (1992): 'Identification de fissures planes par une donnée au bord unique : un procédé direct de localisation et d'identification, C.R. Acad. Sci. Paris 315, série I

[4] Andrieux S., Ben Abda A. (1996): 'Identification of planar cracks by complete overdetermined data : inversion formulae', Inverse Problems 12, 553-563

[5] Ben Abda A., Bui H.D. (2001): 'Reciprocity principle and crack identification in transient thermal problems', Journal of Inverse and ill-posed Problems Vol 9,No1, '1-6'

[6] Brühl M., Hanke M., Pidcock M. (2001): 'Crack detection using electrostatic measurements', M2AN 35, no. 3, 595-605.

[7] Cakoni F., Colton D. (2003): 'The linear sampling method for cracks', Inverse Problems 19, 279-295

[8] Cakoni F., Colton D., Darrigrand E. (2003): 'The Inverse Electromagnetic Scattering Problem for Screens', Inverse Problems 19, 627-642

[9] Cheng J. and Yamamoto M. (2003): 'Uniqueness in an inverse scattering problem within non-trapping polygonal obstacles with at most two incoming waves' Inverse Problems 19 No $6,1361-1384$

[10] Colton D., Kress R. (1992): 'Inverse acoustic and electromagnetic scattering theory', Springer, Berlin

[11] Ikehata M. (1999): 'Enclosing a polygonal cavity in a two-dimensional bounded domain from Cauchy data', Inverse Problems 15, 1231-1241

[12] Kirsch A., Ritter S. (2000): 'A linear sampling method for inverse scattering from an open arc', Inverse Problems 16, 89-105

[13] Kress R. (1995): 'Inverse scattering from an open arc', Math. Meth. Appli. Sci, 18, 267-293

[14] Mönch L. (1997): 'On the inverse scattering problem by an open arc: the sound-hard case', Inverse Problems 15, 1379-1392

[15] Rondi L. (2002): 'Uniqueness for the determination of sound-soft defects in an inhomogeneous planar medium by acoustic boundary measurements', Transactions of the American Mathematical Society,Vol 355,No1, 213-239 


\section{Contents}

\begin{tabular}{|lll}
\hline & Introduction & 3
\end{tabular}

\begin{tabular}{|ll|}
\hline 2 & The acoustic inverse problem
\end{tabular}

$\begin{array}{lll}3 & \text { Some remarks on the scattering problem } & 7\end{array}$

$\begin{array}{llc}4 & \text { Maxwell's equations } & 8\end{array}$

5 Numerical implementation and results $\quad \mathbf{1 2}$

5.1 Numerical difficulties of the inverse scheme . . . . . . . . . . . . . . 12

5.2 Numerical trials . . . . . . . . . . . . . . . . . . . . . . . 13

$\mathrm{RR} \mathrm{n}^{\circ} 5290$ 


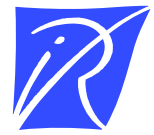

Unité de recherche INRIA Rocquencourt

Domaine de Voluceau - Rocquencourt - BP 105 - 78153 Le Chesnay Cedex (France)

Unité de recherche INRIA Futurs : Parc Club Orsay Université - ZAC des Vignes

4, rue Jacques Monod - 91893 ORSAY Cedex (France)

Unité de recherche INRIA Lorraine : LORIA, Technopôle de Nancy-Brabois - Campus scientifique

615, rue du Jardin Botanique - BP 101 - 54602 Villers-lès-Nancy Cedex (France)

Unité de recherche INRIA Rennes : IRISA, Campus universitaire de Beaulieu - 35042 Rennes Cedex (France)

Unité de recherche INRIA Rhône-Alpes : 655, avenue de l'Europe - 38334 Montbonnot Saint-Ismier (France)

Unité de recherche INRIA Sophia Antipolis : 2004, route des Lucioles - BP 93 - 06902 Sophia Antipolis Cedex (France) 